\author{
By T. D. Kellock, M.D., M.R.C.P. \\ Assistant Physician, Central Middlesex Hospital; Former Research Fellow in Ulcerative Colitis, St. Mark's Hospital
}

Ulcerative colitis is a disease of unknown aetiology, uncertain prognosis and unsatisfactory treatment. In these respects it is, of course, not unique. Its claim to recognition as a clinical entity rests on the existence of a number of cases showing inflammatory changes in the colon and rectum which cannot be attributable to any specific cause. It may be, however, that the cases now grouped together under the diagnosis of ulcerative colitis will turn out to be the end result of a number of different pathological processes or, on the other hand, ulcerative colitis may prove to be a local manifestation of some generalized disease.

\section{The Clinical Picture}

Perhaps the commonest form of the disease is the chronic relapsing type, in which recurrent attacks of diarrhoea are spread over a number of years. The onset of each relapse is often closely related in time to some emotional stress. The length of the relapses is variable, but commonly stretches to several weeks. During the attack there is usually fever and a varying degree of toxaemia. The stools are liquid, containing considerable amounts of mucus and blood, although in a surprising number of cases the latter will be absent to naked eye inspection. Some of the bowel evacuations, which may number up to 20 daily, may consist only of a small amount of blood and mucus, as in dysentery. In many cases one of the most distressing features is the precipitancy and occasional incontinence of faeces. It is not unknown for a patient to refuse hospital admission because of fear of the embarrassment that would be produced by incontinence in the ward.

Pain is not usually a prominent feature of the attacks, being generally slight in character. It is most commonly colicky, preceding movement of the bowels. Exhaustion and weakness are common, as is marked weight loss. There may be a difference of several stone between the weight of a patient during an attack and during remission. A patient in relapse is usually an ill-looking anaemic individual with some fever. The abdomen is soft and may be slightly tender, especially over the descending colon. In the more severe cases ulcera- tion of the mouth is not uncommon. Sigmoidoscopy shows the typical appearance, a bright red $\vec{\sigma}^{\circ}$ friable oedematous mucosa. The normal bloodo vessels are obliterated and the edges of the valves 3 appear rounded because of the oedema instead of $\frac{3}{\omega}$. being of a sharp crescent shape. Spontaneous $\stackrel{\omega}{\omega}$ bleeding is shown by the presence of petechiae and ${ }_{-}$ even the gentlest swabbing produces an ooze of blood. Blood, mucus and frequently pus are 8 present in the lumen. Definite ulceration is rarelyo seen except under magnification. What appears to be an ulcer is usually a plaque of mucus on the $\vec{O}$ wall, which can easily be wiped off. These changes?

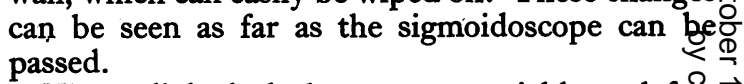

The radiological changes are variable and frê quently show little relationship to the severity af the attack. It is quite possible to have a sevene attack of ulcerative colitis and a barium enenta which shows little departure from normal. In many cases, however, there will be loss of haustra-a tion and a shaggy irregular outline produced byळ the persistence of oedematous islands of mucosa between ulcerated areas. Alternatively (usually ino음 cases with a moderately lengthy history), the typical lead pipe appearance with narrowing, shortening, loss of haustration and a smooth outline. may be seen.

In remission, which may last for many months or even years, the patient may have a normal bowel 3 . habit with one formed stool daily. Sometimes, however, he is prone to looseness. During remission the general health remains excellent, but in a high proportion of cases some sigmoidoscopic abnormality will persist, and it is extremely un $\frac{D}{0}$ common, although it may happen, for a patient who has had an abnormal barium enema to show N complete radiological ' healing.'

\section{Case I}

This man first developed diarrhoea when aged 6r. Ulcerative colitis was diagnosed and he wast admitted to hospital for six weeks. He was then symptom free till the age of 71 , when he had a further attack requiring readmission for eight weeks. Again he remained symptom free until the 
age of 75 , when he was readmitted in extremely poor condition, incontinent of faeces, anaemic and with a temperature rising to I0I. Sigmoidoscopy and $\mathrm{X}$-ray showed gross pseudopolyposis. He made a remarkable spontaneous recovery and has remained completely symptom free for a further period of two years.

In a further proportion of cases the disease shows no such fluctuations, maintaining a continuous course with little change and comparatively little loss of general health, although the diarrhoea may be of such a severe character as to make the patient a social invalid. This is well illustrated by case 2 .

\section{Case 2}

At the age of 25 this engineer suddenly developed diarrhoea with blood and mucus in the stools. There was some improvement after seven weeks in hospital, but the stools remained loose and the bowels were open six times daily. He later developed multiple perianal fistulae and had three further hospital admissions in the next seven years. The diarrhoea continued with 12 to 30 motions daily and he was unable to work for six years on account of the frequency and precipitancy of the bowels, although his general health remained good.

Finally, at the age of 39 , a total colectomy was carried out. In spite of the long period of invalidism he made a good social readjustment and is now in full-time employment which necessitates driving a van.

In marked contrast to this is the acute fulminating type. "This is responsible for a high proportion of the deaths from the disease. It is commoner in the first attack than in relapses and carries a very high mortality rate. The patient is suddenly seized with what appears to be an acute attack of dysentery, except that no specific pathogen can be found in the stool, and there is no response to chemotherapy. The stools are numerous with visible blood and mucus, and the systemic signs are severe. The temperature is high and the pulse rate proportionately higher; the general condition deteriorates rapidly and the patient sinks into a typhoidal state with incontinence, marked dehydration and oedema. Death is due to toxaemia, frequently with generalized peritonitis, following perforation of the nearly gangrenous bowel. The whole disease may last only a few weeks, as shown by the following case.

\section{Case 3}

A woman aged 49 was admitted to a fever hospital with a history of Io days' diarrhoea with the passage of six or seven blood-stained motions daily. A clinical diagnosis of dysentery had been made, but no pathogenic organisms were found. Despite a course of chloramphenicol, her tempera-

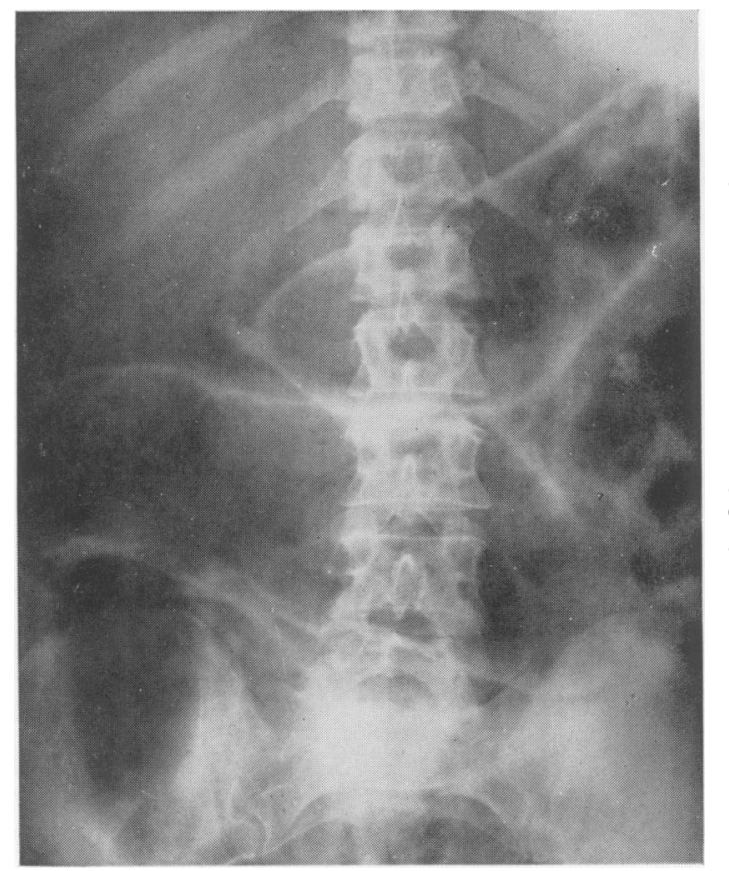

Fig. 1.-Case 3. Plain film of abdomen showing dis tended bowel.

ture ranged from $100^{\circ}$ to $105^{\circ} \mathrm{F}$. She developed abdominal distension and became incontinent of faeces. A plain film of the abdomen showed distended loops of bowel and free air under the diaphragm. Laparotomy revealed generalized peritonitis arising from a perforation in the sigmoid colon. She died four days after operation, the whole illness lasting only 30 days.

Occasionally a similar picture will occur in a relapse, but this is much less common.

\section{Prognosis}

With such a variable clinical picture the prognosis in any individual case is difficult to determine. The mortality rate is highest in the early years of the disease. In a personal series of rog cases, 18 died in their first admission to hospital. This is largely due to cases of the fulminating type, many of whom do not live to have the opportunity of relapsing. Occasionally it happens that a patient makes a complete recovery after an attack of sufficient severity to need hospital treatment.

\section{Case 4}

A man aged 23 was admitted in 1939 for II weeks. When traced in 1954 he stated that not only had he had no recurrence, but he had served 


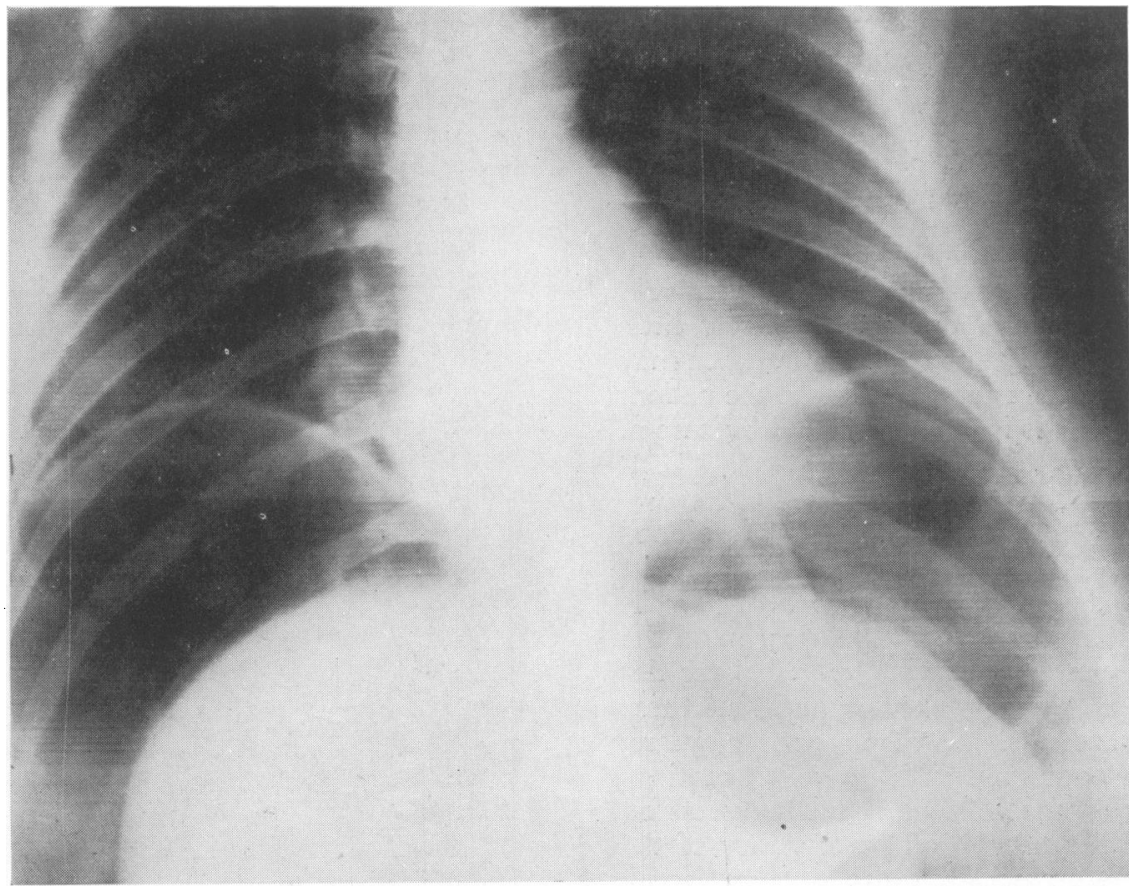

Fig. 2.-Case 3. Plain film showing free gas under the diaphragm.

throughout the Burma campaign and had managed to avoid any attacks of dysentery.

Such cases are uncommon, the vast majority being characterized by remissions. Wheelock and Warren (1955) at the Massachusetts General Hospital found that of 343 cases admitted for this complaint more than Io years previously 45 per cent. were dead of the disease or its complications. Of the survivors, 79 per cent. of those treated medically and 94 per cent. of those treated surgically were well.

In general, the disease seems to progress as it starts, the relapsing case continuing to have similar relapses, and the chronic case persisting.

\section{Complications}

The most serious complication of the disease is perforation of the bowel. It is seen most commonly in the acute fulminating case and is often multiple. This complication, occurring as it does in very ill patients, carries a very high mortality rate. Moreover, in the majority of cases the perforation is silent. It is often impossible to tell from the patient's history, physical examination or temperature chart exactly when perforation occurred. Case 3 above was typical in this respect; the perforation was diagnosed only when free gas was shown under the diaphragm in an X-ray taken to demonstrate the distended bowel. Abdominal dis tension is caused by gas in the dilated atonic diseased bowel, and is of ominous significance, as it frequently announces the onset of perforation.

\section{Case 5}

This 65-year-old woman was admitted with a history of previous diverticulitis and the passage? of a little blood in the stool, but no diarrhoea.

Three days before admission there was a sudden $\frac{0}{3}$ onset of bloody mucoid diarrhoea. In spite of antibiotics, transfusion, etc., her condition deteriorated steadily. One afternoon, six weeks aftero admission, her abdomen became distended; there was no pain, but slight rebound tenderness was noted. Laparotomy the next morning revealed evidence of at least four perforations. Tota $P$ colectomy was performed and after a stormy convalescence she made an excellent recovery. N

Arthritis, usually of the rheumatoid type, is fairly frequent complication. It usually fluctuates with the colitis, improving with remission or colec tomy, but occasionally the joint changes are irreversible.

A number of skin complications may occur Erythema nodosum is moderately frequent in aro acute attack and pyoderma may complicate the 


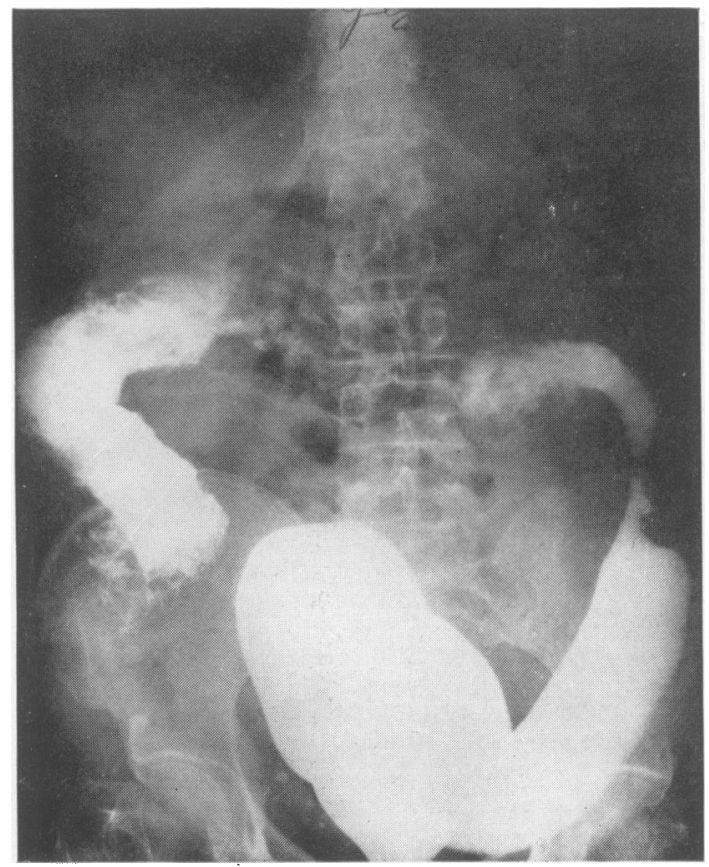

Fig. 3.-Case 6. Barium enema showing stricture at sphenic flexure.

severely ill case. Multiple perianal fistulae may produce a ' pepper-pot' perineum.

Strictures of the rectum and colon usually develop slowly and rarely give rise to obstructive symptoms. They may at times be mistaken for carcinomata.

\section{Case 6}

A woman aged 64 gave a history of colitis for 30 years, which had, however, given no symptoms for the past eight years. When examined at a routine follow-up a mass was felt in the line of the descending colon. Barium enema was reported to show a carcinoma in this region. A stricture was resected and proved to be benign.

One of the surprising features of these strictures is that in many there is little fibrosis, the narrowing being due to enormous thickening of the muscular coat. Whether this is due to hypertrophy of the muscle or is simply the result of longitudinal contraction of the bowel it is difficult to say.

There is abundant evidence that the incidence of carcinoma of the large bowel is increased in cases of long-standing colitis. It is, however, difficult to assess the exact risks as many cases may be undiagnosed, symptoms being attributed to an exacerbation of the colitis. Moreover, the growth may be hard to recognize at operation or post-

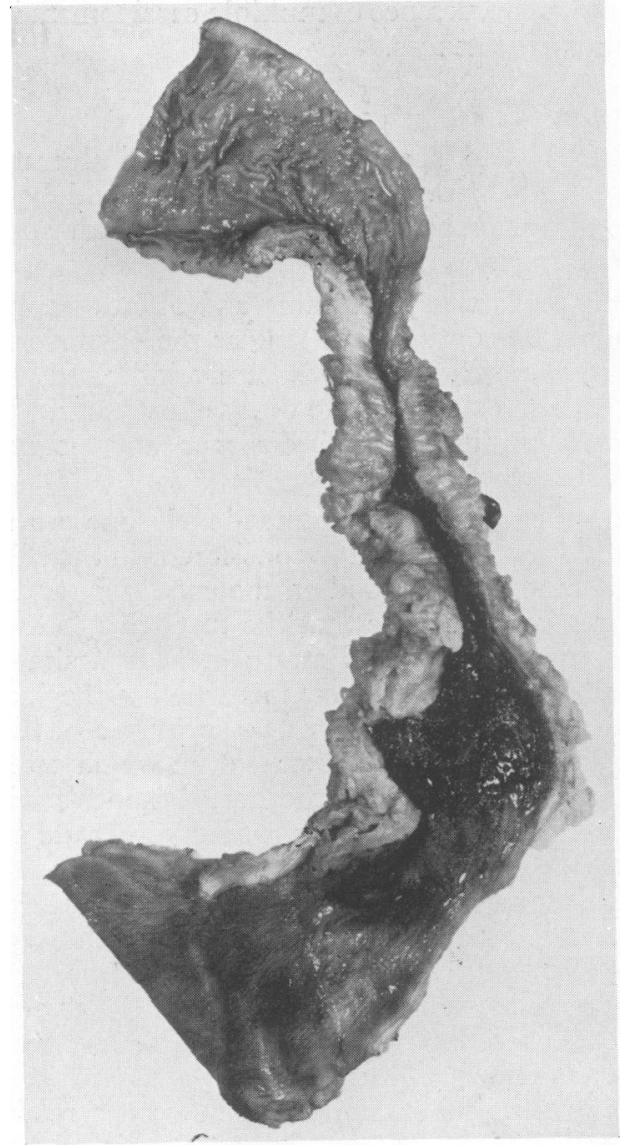

FIG. 4.-Case 6. The resected specimen showing a benign stricture.

mortem, as many present atypical appearances with infiltration by the growth under the mucosa. It is uncommon for malignant changes to take place in cases with less than Io years' history, and the incidence is highest in severe cases, such as those coming to colectomy for chronic disease. Counsell and Dukes (1952) report an incidence of II.I per cent. in resected specimens and Lyons and Garlock (1951) 36 per cent. in cases with a history over 12 years. On the other hand, in 124 cases admitted to the Central Middlesex Hospital only four developed a carcinoma, and in two of these it was the carcinoma that led to the hospital admission. Moreover, $4 \mathrm{I}$ of these cases had a history of more than Io years. As carcinoma of the large bowel is responsible for 3 per cent. of all deaths in the country, it would appear that the increased risk, though real, is not great. The true association of the condition is, however, shown by the high incidence of carcinoma in the younger patients 
and by the occurrence of multiple carcinomata in a number of instances.

\section{Treatment}

There is no specific treatment for ulcerative colitis. The aim of the physician should be to keep the patient as well as possible until spontaneous remission occurs.

Bed rest is always of value and often essential. It should be continued as long as there is fever.

Formal psychotherapy is rarely indicated, but the physician should make every effort to gain the patient's confidence and discover any possible emotional factors.

The diet usually recommended is one with a low residue. There is little consistency in the effect of different items of food on the condition, and in the past it is probable that the diet has been unnecessarily restricted. Extra protein is desirable, and adequate fluids to compensate for the large faecal loss are essential. In the presence of high fever anorexia is often prominent, in which case it will help to supplement the ordinary meals with fortified milk drinks (milk, proteinhydrolysate and glucose). The high loss of potassium justifies the routine use of potassium salts orally, e.g. a mixture of acetate, citrate and bicarbonate, equal parts, $\frac{1}{2}$ g. of each t.d.s. This is of particular importance for all cases receiving cortisone or ACTH, in whom the renal excretion of potassium will be increased. Extra salt may also be needed, but a careful watch must be kept for oedema, which occurs readily in these patients. Blood transfusion is of very great benefit in a high proportion of cases.

The role of antibiotics and chemotherapy is difficult to assess. Each new antibiotic produced is in turn reported to be highly effective in ulcerative colitis, but its use is subsequently found to be disappointing. The reason is that the effect depends on the degree of secondary infection of the diseased bowel by a sensitive organism. In nearly all cases, and especially when there is much pus in the stools, a course of phthalyl sulphathiazole or one of the antibiotics is worth a trial, as the results are occasionally dramatic, if normally disappointing. Streptomycin and penicillin should be given in cases of suspected perforation.

For the milder cases tincture of opium in doses of 5 to 10 minims t.d.s. is of value in diminishing the frequency of the motions and lessening precipitancy. Occasionally hexamethonium is helpful in diminishing bowel motility, but usually the sideeffects from an adequate dose are as troublesome as the bowel symptoms and there is a real risk of producing ileus. In some cases 'Isogel' may be of help in forming a soft homogeneous motion. This is particularly so in those cases who pass one rather hard stool daily and a number of others consisting of only blood, serum and mucus.

Truelove and Witts (1954) reported the results $\stackrel{C}{.}$ of a therapeutic trial of cortisone in this condition. Conflicting reports on the value of this hormone? had been published previously. The trial organized등 by the Medical Research Council was blind, $\frac{\overline{\bar{c}}}{\overline{\mathrm{N}}}$ neither the patient nor the physician knowing whether the hormone or a dummy tablet was being administered. It was found that $4 \mathrm{I}$ per cent. of thew cortisone group were in remission at the end of six $\overrightarrow{0}$ weeks' treatment, but only 16 per cent. of thecontrol group. The effect was more marked in $\vec{\sigma}$ first attacks than in relapses.

The effect of cortisone or of ACTH is most marked on the toxaemia. There is a rapid gain in weight, the temperature may fall abruptly, but the ${ }_{-}^{\omega}$ pulse rate usually comes down more slowly. There is often little effect initially on the diarrhoea and. it is rare for much change to occur in the sig-co moidoscopic or radiological findings for severa $b$ weeks. The dangers of silent perforation under the influence of these hormones have been much $\vec{O}$ stressed, but, as has been pointed out above, silent? perforation may occur anyway, and it does noto seem that the use of cortisone increases this risk greatly. Hormone therapy sometimes fails to poi $\overrightarrow{0}$ duce change in the patient's condition, may not arrest rapid deterioration and in many mild cases is unnecessary. Indications for this form of therapyo have not yet been worked out. It would appear to be most useful in acute toxic cases (particularlyo first attacks) who are not so ill that emergencyo surgery may be necessary in the next few days, and also in those cases where a patient with a shorto history has a moderately severe relapse and shows no evidence of improving or deteriorating after af few weeks, i.e. cases not ill enough to warrant surgery, but not well enough for discharge froms hospital. In these hormone therapy may induce $\frac{a}{0}$. remission.

\section{Surgical Treatment}

As operation in ulcerative colitis involves the construction of a permanent ileostomy in nearly alp cases, it is not to be considered lightly. The indications for operation are (I) to save life, usually. in the acute fulminating type of case; (2) fots chronic invalidism. The risk of carcinoma, while real, is in itself not enough to justify radicaR surgery, but will be a deciding factor in some
borderline cases with a long history.

The operation of choice is ileostomy and sub운 total colectomy as a first stage with subsequen excision of the rectum. In the few cases with a normal rectum ileorectal anastomosis may be possible.

The results of surgery are extremely satisfactory 
and since the introduction of the modern adhesive appliances life with an ileostomy is never as much of a handicap as the patient fears.

\section{Summary}

Ulcerative colitis is a disease of unknown aetiology characterized by friability of the colonic mucosa leading to ulceration.

The clinical course is extremely varied, but is usually characterized by remissions and relapses. The initial attack carries the highest mortality rate.

Medical treatment aims at supporting the patient over an attack until remission occurs. The use of cortisone or ACTH for this purpose is indicated in suitable cases.

Ileostomy and colectomy is indicated for the severe toxic attack which appears likely to be fatal, and for chronic invalidism in the long-standing case.

\section{BIBLIOGRAPHY}

COUNSELL, P. B., and DUKES, C. E. (1952), B. F.S., 39, 198, I. LYONS, A. S., and GARLOCK, J. H. (195I), Gastroent., 18, 178. TRUELOVE, S. C., and WITTS, L. J. (1954), Brit. med. f., ii, 375.

WHEELOCK, F. C., and WARREN, R. (1955), New Eng. F. Med., 252, 421 .

\title{
TUBERCULOSIS OF THE SPINE
}

\section{A Reassessment of the Problem and the Results of Conservative Treatment}

\author{
By A. J. HaRrold, F.R.C.S. \\ Orthopaedic Registrar, St. Mary's Hospital, London
}

In the years 1947 to $195 \mathrm{I}$ there were 224 admissions for tuberculosis of the spine to Heatherwood Orthopaedic Hospital, Ascot. The material for this paper has been collected from their case records to show the present behaviour of the disease when managed along conservative lines.

Spinal caries in England is a much more benign disease than it was 50 years ago. Information about its response to conventional treatment is important in assessing the value of modern surgical and chemotherapeutic measures.

\section{TABLE I}

Patients with active disease of vertebral body . $\quad 192$

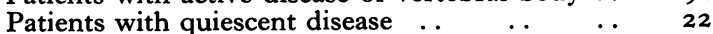

Patients with quiescent disease but sinuses $\quad \ldots \quad 7$

Patients with disease of spinous process only $\quad \ldots \quad 2$

Patients with disease of pedicle only $\ldots \quad \ldots$ I $^{2}$

\section{Material}

An analysis of the 224 admissions is given in Table $\mathrm{I}$. The cases to be considered in detail are the 192 with active disease of the vertebral body. These may be divided on clinical grounds into 146 fresh cases without a previous full course of treatment for the spinal lesion, and 46 cases of recurrence. The 192 admissions concern 177 patients, as 13 were admitted twice and one three times in the period under review.

\section{Incidence}

The age and sex incidence are set out in Graph $\mathrm{I}$ and Table 2 respectively. Graph $I$ is in keeping

\section{TABLE 2.-SeX INCIDENCE}

$\begin{array}{lccccr} & & & & \text { Male } & \text { Female } \\ \text { Fresh cases } & . & \ldots & \ldots & \text { 61 }(42 \%) & 85(58 \%) \\ \text { Reactivated cases } & \ldots & \ldots & 22(48 \%) & 24(52 \%) \\ \text { Total .. } & . . & \ldots & . . & 83(43 \%) & \text { 109 }(57 \%)\end{array}$

with the findings of most modern authorities (e.g. Girdlestone and Somerville, 1952; Dobson, 1951) that tuberculosis of the spine is no longer a disease of childhood but affects mainly young adults.

The incidence of disease at different levels of the spine is shown in Graph 2. Cervical caries $5^{\circ}$ years ago was said to occur in about one case in seven (Keetley, 1900). In the present series it forms less than I per cent. Two reasons for this are suggested. The diminished frequency of spinal tuberculosis in children may be one factor, for Dobson (I95I) has shown that cervical caries is proportionately more common in children than adults. Secondly, the decline may be linked with the improved control of tuberculous cervical lymphadenitis with less chance of local spread of infection to the vertebral column at this level.

Analysis of the fresh cases shows that tuberculosis is progressively more common from the top to the bottom of the spine, although re-activated disease is seen most often at the lower thoracic 